\title{
Gyroid Optical Metamaterials: Calculating the Effective Permittivity of Multidomain Samples
}

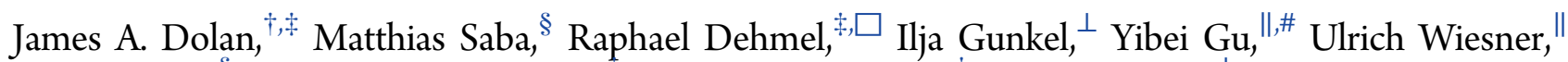
Ortwin Hess, ${ }^{\S}$ Timothy D. Wilkinson, ${ }^{\dagger}$ Jeremy J. Baumberg, ${ }^{\ddagger}$ Ullrich Steiner, ${ }^{\perp}$ and Bodo D. Wilts ${ }^{*, \perp}$

${ }^{\dagger}$ Department of Engineering, University of Cambridge, J.J. Thomson Avenue, Cambridge CB3 OFA, U.K.

${ }^{\ddagger}$ Nanophotonics Centre, Department of Physics, University of Cambridge, J.J. Thomson Avenue, Cambridge CB3 0HE, U.K.

${ }^{\S}$ Department of Physics, Imperial College, Prince Consort Road, London SW7 2BB, U.K.

${ }^{\perp}$ Adolphe Merkle Institute, Chemin des Verdiers 4, 1700 Fribourg, Switzerland

"Department of Materials Science and Engineering, Cornell University, 330 Bard Hall, Ithaca, New York 14853-1501, United States

Supporting Information

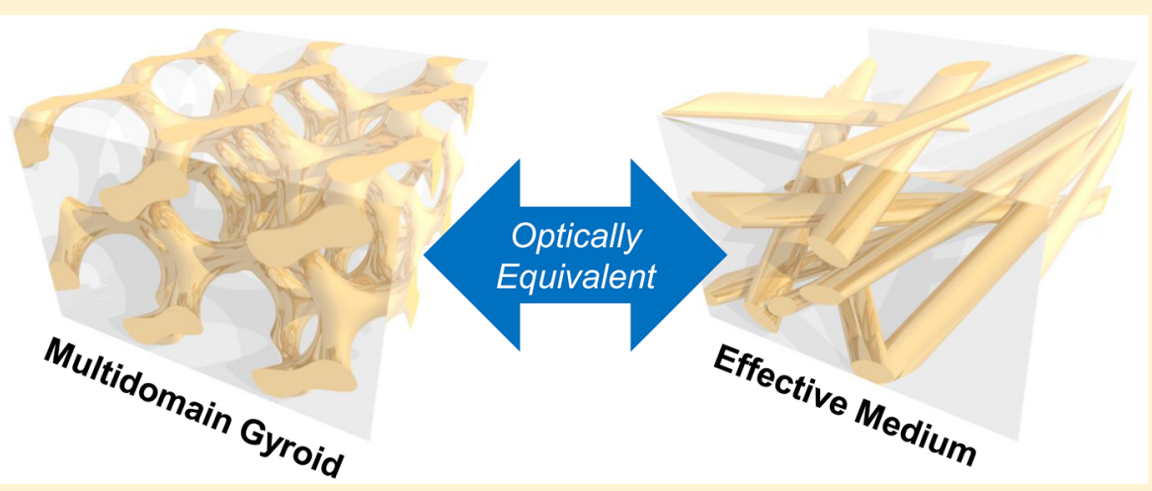

ABSTRACT: Gold gyroid optical metamaterials are known to possess a reduced plasma frequency and linear dichroism imparted by their intricate subwavelength single gyroid morphology. The anisotropic optical properties are, however, only evident when a large individual gyroid domain is investigated. Multidomain gyroid metamaterials, fabricated using a polyisoprene- $b$-polystyrene- $b$-poly(ethylene oxide) triblock terpolymer and consisting of multiple small gyroid domains with random orientation and handedness, instead exhibit isotropic optical properties. Comparing three effective medium models, we here show that the specular reflectance spectra of such multidomain gyroid optical metamaterials can be accurately modeled over a broad range of incident angles by a Bruggeman effective medium consisting of a random wire array. This model accurately reproduces previously published results tracking the variation in normal incidence reflectance spectra of gold gyroid optical metamaterials as a function of host refractive index and volume fill fraction of gold. The effective permittivity derived from this theory confirms the change in sign of the real part of the permittivity in the visible spectral region (so, that gold gyroid metamaterials exhibit both dielectric and metallic behavior at optical wavelengths). That a Bruggeman effective medium can accurately model the experimental reflectance spectra implies that small multidomain gold gyroid optical metamaterials behave both qualitatively and quantitatively as an amorphous composite of gold and air (i.e., nanoporous gold) and that coherent electromagnetic contributions arising from the subwavelength gyroid symmetry are not dominant.

KEYWORDS: gyroid, optical metamaterial, effective medium model, block copolymer, polymer self-assembly

E lectromagnetic metamaterials are artificially structured materials for which the electromagnetic response is a function of not only their chemical composition but also their structure. ${ }^{1}$ When the length scales of the constituent structural units are significantly smaller than the wavelength of interest, the response of the metamaterial may be described by the homogenized effective permittivity $\varepsilon_{\text {eff }}(\omega)$ and permeability $\mu_{\text {eff }}(\omega)$. Metamaterials greatly expand the range of electromagnetic responses otherwise found in natural and synthetic bulk materials. They therefore offer huge flexibility to exploit exotic situations, or spatial arrangements of effective permittivity and permeability in electromagnetic material design. Potential applications famously include cloaking devices and "superlenses". 2,3 However, of perhaps more immediate technological interest is the access to particularly large and small, or strongly anisotropic (e.g., hyperbolic), material parameters and their associated science and engineering. ${ }^{4}$

Whereas metamaterials that operate in the microwave regime have existed for decades, it is only relatively recently that

Received: June 14, 2016

Published: September 6, 2016 
advances in nanofabrication have allowed the manufacture of optical metamaterials. ${ }^{5}$ Due to the requirement that the structural units of a metamaterial be deeply subwavelength, ideally by an order of magnitude or more, optical metamaterials demand feature sizes on the length scale of tens of nanometers. However, many early top-down lithographic techniques either were intrinsically two-dimensional (e.g., a single material layer) or ventured into the third dimension only by tedious and nonscalable stacking of individual layers. These techniques are arguably still incapable of fabricating three-dimensional nanostructures over macroscopically large areas with sufficient speed and cost-effectiveness to allow optical metamaterials to attain true technological relevance. Therefore, until recently, three-dimensional optical metamaterials presented a considerable challenge. ${ }^{6}$

Polymer self-assembly presents an alternative and particularly intriguing means by which to fabricate intricate one-, two-, and three-dimensional morphologies on the appropriate length scales, from which optical metamaterials may be replicated. ${ }^{7}$ Block copolymers are macromolecules consisting of two or more covalently linked homopolymers ("blocks") that can microphase separate into various well-defined morphologies due to the chemical dissimilarity between blocks. In block copolymers consisting of three distinct and sequential blocks (i.e., linear triblock terpolymers) one such morphology is the alternating gyroid: two single gyroid networks, each composed of one block, separated by a matrix of the third. The single gyroid is a continuous, chiral, and triply periodic cubic network with a constant mean curvature surface that is found in a range of natural and synthetic self-assembled systems. ${ }^{8}$ As the two single gyroid networks in the alternating gyroid are chemically distinct, it is possible to selectively remove just one such network and use the remaining voided terpolymer as a sacrificial template for metal deposition. ${ }^{9,10}$ It was using this technique that Vignolini et al. fabricated the first truly threedimensional self-assembled optical metamaterial: a gold single gyroid with a $\sim 50 \mathrm{~nm}$ unit cell size and $30 \%$ fill fraction. ${ }^{11}$

Gyroid metamaterials possess a range of optical properties not exhibited by the pure metal, including an unambiguous optical anisotropy. ${ }^{8}$ Large individual domains exhibit a significantly decreased plasma frequency and linear dichroism, evidenced by the shift of the extinction peak by up to $100 \mathrm{~nm}$ upon rotation under linearly polarized light. ${ }^{11}$ Both the plasma frequency and the linear dichroism are additionally a function of the unit cell size, fill fraction, and dielectric environment. ${ }^{12,13}$ Although much is now known about the optical properties of gyroid metamaterials, suitable estimates of the effective material parameters remain elusive, thereby hindering a true appraisal of the metamaterial's potential utility. A trihelical metamaterial (THM) model of the gyroid as periodic disconnected helices allows the analytical derivation of the permittivity, permeability, and chiral coupling parameter. ${ }^{14,15}$ From these the plasma frequency of the metamaterial and its variation with unit cell size, fill fraction, and dielectric environment can be successfully predicted. ${ }^{12}$ However, the THM model does not describe the linear dichroism of gyroid metamaterials, and the model is unsuitable to average over an ensemble of small domains of random orientation and handedness. It is therefore of great interest to correctly identify the effective material parameters of gyroid metamaterials and to understand to what extent the ordered subwavelength structure continues to affect the optical response when the macroscopic order is not long range.
In this work, we investigate the optical properties of multidomain gold gyroid optical metamaterials fabricated from the alternating gyroid phase of a polyisoprene- $b$ polystyrene- $b$-poly(ethylene oxide) (ISO) triblock terpolymer. The specular reflection of the isotropic metamaterial is measured under a range of angles of incidence of light, and the resulting reflectance spectra are compared to those predicted by three of the simplest (and therefore most common) analytical effective medium theories applicable to amorphous composites of spherical or ellipsoidal inclusions of one material in a host matrix of another. We show that a Bruggeman model with ellipsoidal inclusions is the only model considered that can reproduce the features present in the measured reflectance spectra of the investigated multidomain gold gyroid optical metamaterials and that such optical metamaterials behave substantially similarly to nanoporous gold.

\section{EFFECTIVE MEDIUM THEORIES}

For a material that is homogeneous at a particular length scale, its physical properties may be described by a reduced set of effective material parameters. ${ }^{16}$ Effective medium theories are approximate analytical means to ascribe a homogeneous (although potentially anisotropic) material parameter, in this case $\varepsilon_{\text {eff }}(\omega)$, to a heterogeneous mixture of two or more components. ${ }^{1}$ Such theories are anticipated to hold when the inhomogeneity length scale of the material and the local variation in the electric field are both significantly smaller than the wavelength of interest. ${ }^{17}$ Although numerous approaches of varying complexity exist, the two considered in this work are arguably the most prevalent theories: the Maxwell-Garnett theory for spherical inclusions and the Bruggeman theory. ${ }^{1,17}$

Maxwell-Garnett Theory: Case of Spherical Inclusions. The simplest form of the Maxwell-Garnett effective medium theory considers spherical inclusions of one material in a host matrix of another material (Figure 1a). The effective permittivity is found by solving the following implicit equation: ${ }^{1}$

$$
\begin{aligned}
& \frac{\left(\varepsilon_{1}(\omega)-\varepsilon_{2}(\omega)\right) f_{1}}{\varepsilon_{2}(\omega)+\left(\varepsilon_{1}(\omega)-\varepsilon_{2}(\omega)\right) A} \\
& +\frac{\varepsilon_{2}(\omega)-\varepsilon_{\text {eff }}(\omega)}{\varepsilon_{2}(\omega)+\left(\varepsilon_{\text {eff }}(\omega)-\varepsilon_{2}(\omega)\right) A}=0
\end{aligned}
$$

where $\varepsilon_{\text {eff }}(\omega)$ is the (isotropic) effective permittivity of the composite, $\varepsilon_{1}(\omega)$ is the permittivity of the first component (the inclusion), $\varepsilon_{2}(\omega)$ is the permittivity of the second component (the host), $f_{1}$ is the volume fraction of the first component, $A$ is the depolarization factor (1/3 for spherical inclusions), and $\omega$ is frequency. The depolarization factor relates the dipole moment of a dielectric ellipsoid to the applied external electric field and varies from 0 (disks) to 1 (wires). The above theory is strictly valid only when the volume fill fraction of the inclusions is small. As a result, the above equation is not invariant under the transformation $\varepsilon_{1} \leftrightarrow \varepsilon_{2}$ and $f_{1} \leftrightarrow f_{2}$. Asymmetric formulations such as this are only valid for low volume fractions of the polar phase (see, for example, Table 1 in ref 18). Nonetheless, the Maxwell-Garnett effective medium theory should be considered due to its widespread usage and having previously been employed to justify the differing behavior of gold gyroid metamaterials from an amorphous composite. ${ }^{11}$ 
a)

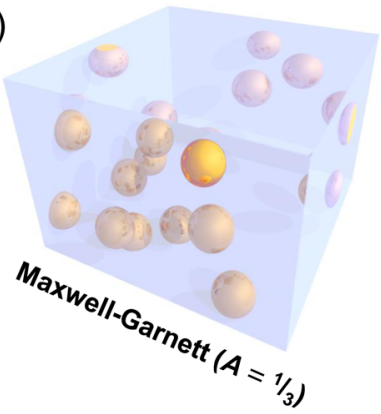

c)

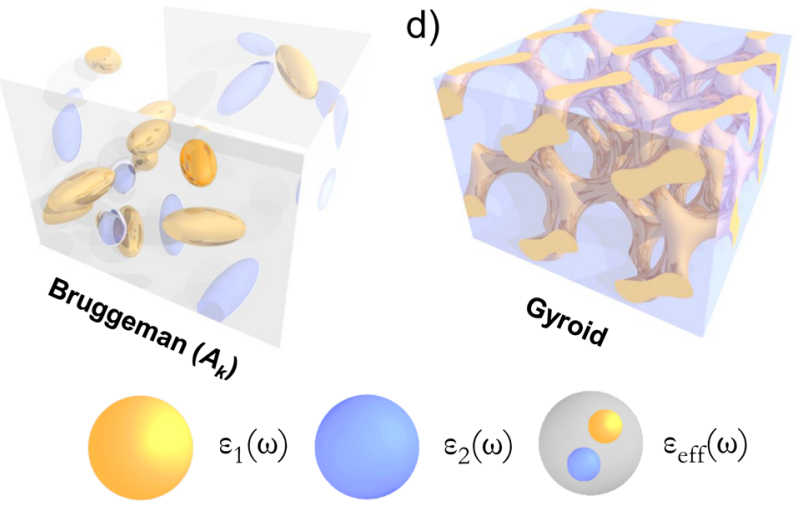

b)

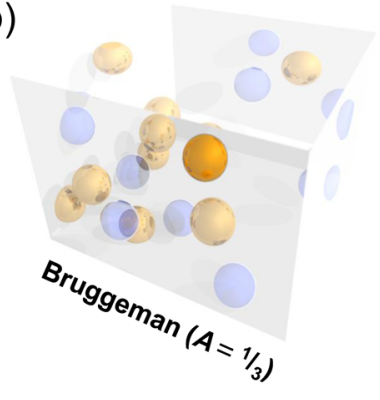

Figure 1. Sketches of the mathematical representations of various effective medium theories. (a) Maxwell-Garnett, eq 1, where the effective permittivity $\left(\varepsilon_{\text {eff }}\right)$ is derived by considering spherical inclusions $(A=1 / 3)$ of one material $\left(\varepsilon_{1}\right)$ in a host matrix of another $\left(\varepsilon_{2}\right)$. (b) Bruggeman $(A=1 / 3)$, eq 2 , where the effective permittivity $\left(\varepsilon_{\text {eff }}\right)$ is derived by averaging over single inclusions $\left(\varepsilon_{1}\right.$ and $\left.\varepsilon_{2}\right)$ individually embedded in an effective medium consisting of all other inclusions $\left(\varepsilon_{\text {eff }}\right)$. (c) Bruggeman $\left(A_{k}\right)$, eq 3 , where the effective permittivity $\left(\varepsilon_{\text {eff }}\right)$ is derived analogously to the case where $A=1 / 3$ except that all individual spherical inclusions are replaced by randomly oriented ellipsoids. (d) The single gyroid morphology for which an effective medium theory is sought.

Bruggeman Theory: Case of Spherical Inclusions. We adopt here a version of the Bruggeman effective medium theory, in which all constituents of the composite are treated symmetrically. If the inclusions are again assumed to be spherical (Figure 1b), the effective permittivity is found by solving the following implicit equation (see Supporting Information for more information): ${ }^{18}$

$$
\begin{aligned}
& \frac{\left(\varepsilon_{1}(\omega)-\varepsilon_{\text {eff }}(\omega)\right) f_{1}}{\varepsilon_{\text {eff }}(\omega)+\left(\varepsilon_{1}(\omega)-\varepsilon_{\text {eff }}(\omega)\right) A} \\
& +\frac{\left(\varepsilon_{2}(\omega)-\varepsilon_{\text {eff }}(\omega)\right) f_{2}}{\varepsilon_{\text {eff }}(\omega)+\left(\varepsilon_{2}(\omega)-\varepsilon_{\text {eff }}(\omega)\right) A}=0
\end{aligned}
$$

where $\varepsilon_{\text {eff }}(\omega), \varepsilon_{1}(\omega), \varepsilon_{2}(\omega), f_{1}, A$, and $\omega$ are defined as above, and $f_{2}=1-f_{1}$ is the volume fill fraction of the second component. Note that the above equation is now invariant under the transformation $\varepsilon_{1} \leftrightarrow \varepsilon_{2}$ and $f_{1} \leftrightarrow f_{2}$, indicating that the Bruggeman effective medium theory is applicable for any given volume fill fraction. It is therefore anticipated that this simplest form of the Bruggeman theory may more accurately model the behavior of $30 \%$ fill fraction gyroid metamaterials, such as those considered here.

Bruggeman Theory: Case of Ellipsoidal Inclusions. Although the Bruggeman effective medium theory is insensitive to the absolute size of the subwavelength inclusions of a composite, it is possible to extend the theory to take anisotropy of the inclusions into account. This is done by modeling such inclusions as aligned ellipsoids with semiaxes $a_{k}$. In such a case the depolarization factor $A$ in eq 2 is replaced by

$$
A_{k}=\frac{a_{1} a_{2} a_{3}}{2} \int_{0}^{\infty} \frac{\mathrm{d} u}{\left(a_{k}^{2}+u\right) \sqrt{\left(a_{1}^{2}+u\right)\left(a_{2}^{2}+u\right)\left(a_{3}^{2}+u\right)}}
$$

where $A_{k}$ is the depolarization factor for light linearly polarized along the $k$ th axis of an aligned ellipsoidal inclusion. Note that the derived effective permittivity is hence only valid for that same relative orientation of linearly polarized light and the effective permittivity of the material is anisotropic (i.e., when all ellipsoidal inclusions are assumed to be aligned). However, it is also possible to define a macroscopically isotropic effective permittivity while allowing for microscopic anisotropy if the ellipsoidal inclusions are assumed to be randomly oriented in the composite (Figure 1c). In such a case, the effective permittivity is derived by solving the following implicit equation (see Supporting Information):

$$
\begin{aligned}
& \sum_{k=1}^{3} \frac{\left(\varepsilon_{1}(\omega)-\varepsilon_{\text {eff }}(\omega)\right) f_{1}}{\varepsilon_{\text {eff }}(\omega)+\left(\varepsilon_{1}(\omega)-\varepsilon_{\text {eff }}(\omega)\right) A_{k}} \\
& \quad+\frac{\left(\varepsilon_{2}(\omega)-\varepsilon_{\text {eff }}(\omega)\right) f_{2}}{\varepsilon_{\text {eff }}(\omega)+\left(\varepsilon_{2}(\omega)-\varepsilon_{\text {eff }}(\omega)\right) A_{k}}=0
\end{aligned}
$$

where $\varepsilon_{\text {eff }}(\omega), \varepsilon_{1}(\omega), \varepsilon_{2}(\omega), f_{1}, f_{2}$, and $\omega$ are as defined above and $A_{k}$ are the three depolarization factors corresponding to the geometry of the ellipsoidal inclusions $\left(A_{1}+A_{2}+A_{3}=1\right)$. Note that this equation is identical for both a polycrystalline sample consisting of numerous randomly oriented anisotropic domains and a composite sample consisting of equal amounts of three isotropic constituents of differing permittivities or depolarization factors. ${ }^{16}$ Note also that the Maxwell-Garnett theory may similarly be extended to take into account microscopic anisotropy (vide infra).

\section{RESULTS}

An optical and electron micrograph of the multidomain gyroid metamaterial, the geometry of the experiment, and the resulting reflectance spectra for transverse magnetic (TM) and transverse electric (TE) polarizations of incident light are shown in Figure 2. The optical micrograph captured under linearly polarized light (Figure 2a) highlights the light orange color of the metamaterial, and the electron micrograph of an individual domain (Figure $2 \mathrm{~b}$ ) is consistent with the gyroid nanostructure previously observed for this terpolymer. ${ }^{11-13}$ The small size and multiplicity of the domains can be more clearly seen in Figure S1. Both TM (Figure 2d) and TE (Figure 2e) reflectance spectra are qualitatively similar to those of bulk gold (Figure S2), insofar as they exhibit low reflectance at shorter wavelengths and increased reflectance at longer wavelengths across all angles of incidence. (Note that in Figures 2-5, reflectance spectra are plotted on a log scale as they exhibit spectral features that span multiple orders of magnitude.) In bulk gold, these features are attributable to interband transitions and the plasma frequency, respectively. However, the measured spectra differ quantitatively from those of bulk gold due to both a lowered effective plasma frequency and their variation with angle of incidence. Otherwise, the spectra exhibit the general decrease (increase) in reflectance for 


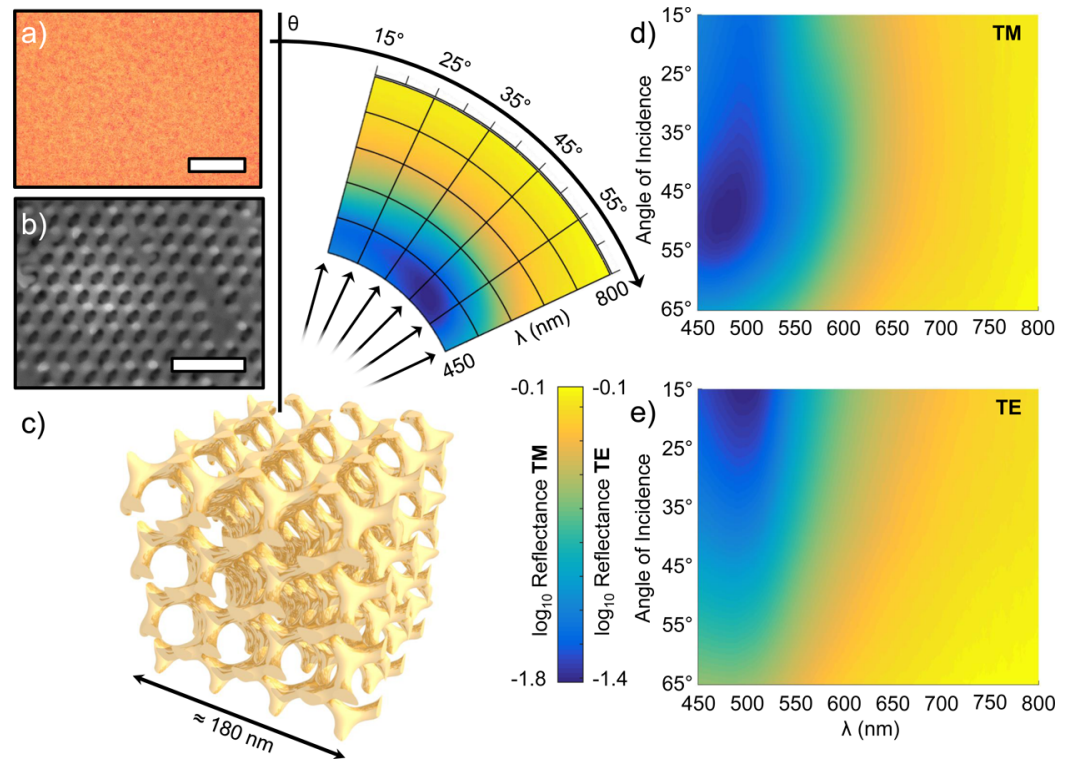

Figure 2. Gyroid optical metamaterials: optical and electron micrographs, experiment geometry, and measured reflectance spectra. (a) Optical micrograph of multidomain gold gyroid metamaterial under linearly polarized light (scale bar $100 \mu \mathrm{m}$ ). (b) Electron micrograph of an individual domain of the sample (scale bar $200 \mathrm{~nm}$ ). (c) Specular reflectance spectra of the gyroid metamaterial are measured as a function of angle of incidence between $15^{\circ}$ and $65^{\circ}$ and 450 and $800 \mathrm{~nm}$. Although a single gyroid domain is shown for clarity, the measurement spot size in fact encompasses a large number of randomly oriented gyroid domains. (d) Reflectance spectra as a function of angle of incidence for transverse magnetic (TM) polarized light (log color scale extends from -1.8 to -0.1). (e) The same for transverse electric (TE) polarized light (log color scale extends from -1.8 to -0.1$)$.
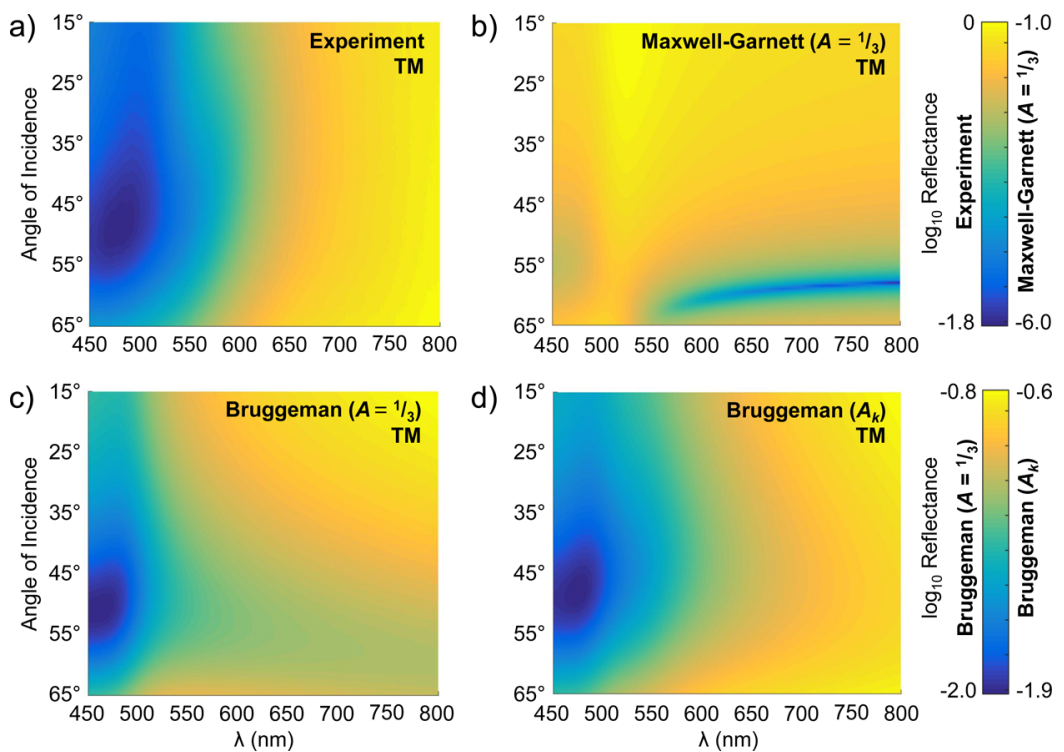

Figure 3. Comparison of measured and modeled TM reflectance spectra as a function of angle of incidence using three effective medium theories. (a) Measured reflectance spectra for transverse magnetic (TM) polarized light repeated from Figure $2 \mathrm{~d}(\log$ color scale extends from -1.8 to 0 ). (b) Reflectance spectra generated using the Maxwell-Garnett theory (log color scale extends from -6.0 to -1.0$)$. (c) The same generated using the Bruggeman theory and $A=1 / 3$, i.e., spherical inclusions (log color scale extends from -2.0 to -0.8 ). (d) The same generated using the Bruggeman theory and $A_{1}=0.66, A_{2}=0.34$, and $A_{3}=0$, i.e., ellipsoidal inclusions ( $\log$ color scales extends from -1.9 to -0.6 ).

TM (TE) polarized light with increasing angle of incidence, with an effective Brewster's angle of $\sim 50^{\circ}$.

A comparison between the measured and modeled reflectance spectra across all wavelengths and angles of incidence for TM polarized light is shown in Figure 3; the same for TE polarized light is shown in Figure S3. The effective permittivity $\varepsilon_{\text {eff }}(\omega)$ used to generate the modeled spectra in Figure $3 \mathrm{~b}$ was calculated using the Maxwell-Garnett theory by setting $\varepsilon_{1}(\omega)=\varepsilon_{\mathrm{Au}}(\omega), \varepsilon_{2}(\omega)=1, f_{1}=0.3$, and $A=1 / 3$ (i.e., spherical inclusions) in eq 1. Clearly the Maxwell-Garnett effective medium theory is a poor model for the effective permittivity of the gyroid metamaterial, as it predicts a striking dip in reflectance at an effective Brewster's angle of $\sim 60^{\circ}$, which is entirely absent in the measured data. Despite also assuming spherical inclusions, the effective permittivity calculated using the Bruggeman theory, by again setting $\varepsilon_{1}(\omega), \varepsilon_{2}(\omega), f_{1}$, and $A$ as above in eq 2 , results in a far better qualitative match between the measured and modeled 
a)

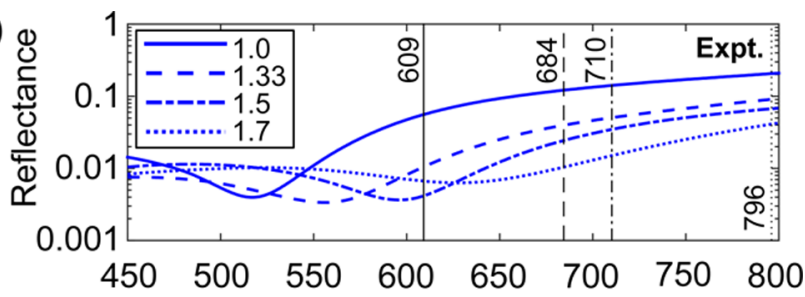

b)

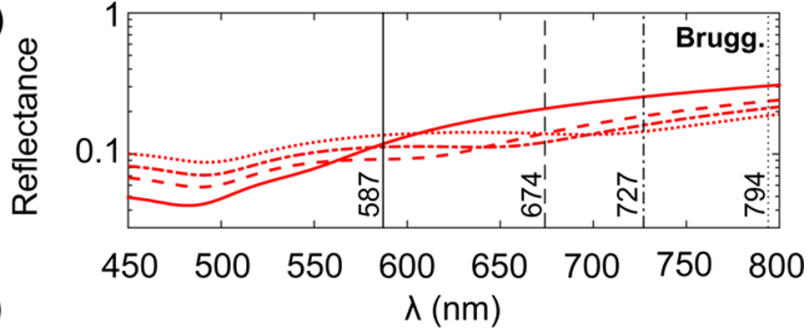

c)

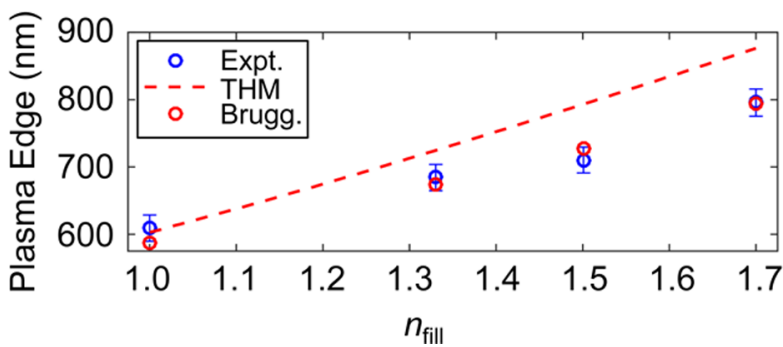

Figure 4. Variation in normal incidence reflectance spectra and plasma edge as a function of host refractive index. (a) Measured reflectance spectra at normal incidence for $30 \%$ fill fraction gold gyroid metamaterials infiltrated with media of refractive indices 1.0 (solid line), 1.33 (dashed line), 1.5 (dotted-dashed line), and 1.7 (dotted line) and the associated plasma edge of each spectrum in nanometers (labeled vertical lines of the same style). Data are reproduced from Salvatore et al. ${ }^{12}$ (b) Modeled normal incidence reflectance spectra using the optimized Bruggeman model where $\varepsilon_{2}(\omega)$ of eq 3 is set to $1.0^{2}$ (solid line), $1.33^{2}$ (dashed line), $1.5^{2}$ (dotted-dashed line), and $1.7^{2}$ (dotted line) and the associated plasma edge of each spectrum in nanometers (labeled vertical lines of the same style). (c) Plasma edge against refractive index of host for measured (Expt.) and modeled (Brugg.) spectra and the relationship predicted by the trihelical metamaterial (THM) model, reproduced from Salvatore et al. ${ }^{12}$

reflectance spectra (Figure 3c). The global reflectance minimum at $\sim 50^{\circ}$ between 450 and $500 \mathrm{~nm}$ is reproduced well. However, the spectra still exhibit a decrease in reflectance at long wavelengths and high angles of incidence, which is not present in the experimental data. This decrease in reflectance is absent in the reflectance spectra of Figure 3d, which uses our microscopically anisotropic Bruggeman theory. These spectra were modeled by setting $\varepsilon_{1}(\omega), \varepsilon_{2}(\omega)$, and $f_{1}$ as above and $A_{1}=$ $0.66, A_{2}=0.34$, and $A_{3}=0$ in eq 3 . These depolarization factors were found by performing a constrained optimization to fit the TM reflectance spectra generated by the model to the measured data (see Experimental Methods).

A quantitative comparison of the goodness of fit of each model is presented in Table 1. Cost functions $f(\mathbf{A})$ (see Experimental Methods) are tabulated not only for TM but also for TE polarization using the depolarization factors derived from the TM measurements. Note that the cost function evaluated for TE polarization using the depolarization factors derived from the TM measurements $\left(A_{1}=0.66, A_{2}=0.34\right.$, and $A_{3}=0$ ) is only $0.66 \%$ larger than the same cost function evaluated for TE polarization using the depolarization factors derived from the TE measurements $\left(A_{1}=0.70, A_{2}=0.30\right.$, and a)

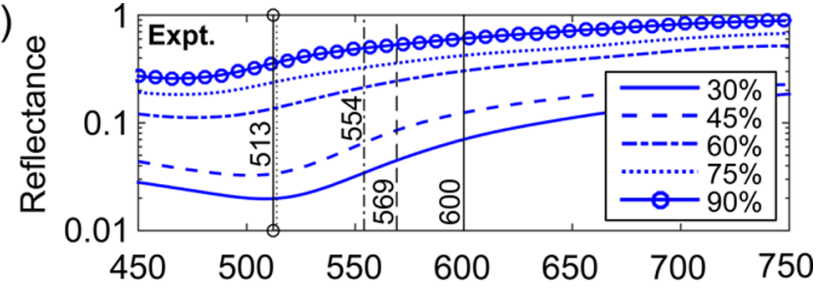

b)

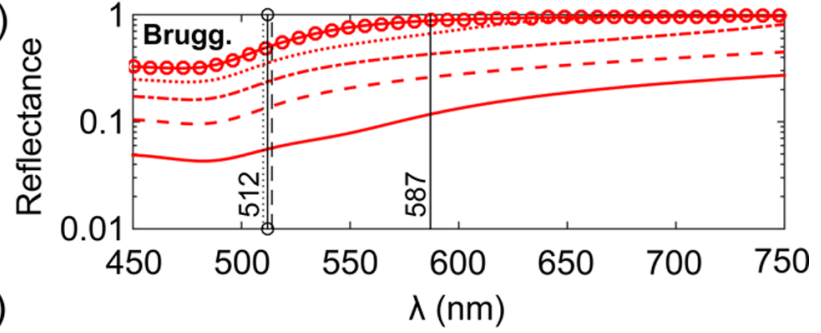

c)

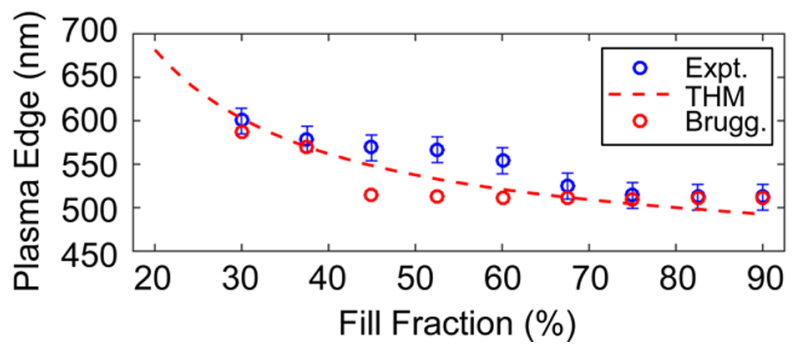

Figure 5. Variation in normal incidence reflectance spectra and plasma edge as a function of gold volume fill fraction. (a) Measured normal incidence reflectance spectra of gold gyroid metamaterials with volume fill fractions of $30 \%$ (solid line), $45 \%$ (dashed line), $60 \%$ (dotteddashed line), $75 \%$ (dotted line), and 90\% (solid line with circles) and the associated plasma edge of each spectrum in nanometres (labeled vertical lines of the same style). Data reproduced from Salvatore et al. ${ }^{12}$ (b) Modeled normal incidence reflectance spectra using the optimized Bruggeman model where $f_{1}$ of eq 3 is set to 0.30 (solid line), 0.45 (dashed line), 0.60 (dotted-dashed line), 0.75 (dotted line), and 0.90 (solid line with circles) and the associated plasma edge of each spectrum in nanometers (labeled vertical lines of the same style). (c) Plasma edge against volume fill fraction of gold for measured (Expt.) and modeled (Brugg.) spectra and the relationship predicted by the trihelical metamaterial (THM) model, reproduced from Salvatore et al. $^{12}$

Table 1. Table of Cost Functions $f(A)$ (See Experimental Methods) Associated with the Various Effective Medium Theories (model), Equations (eq), Polarizations (pol), and Vector of Depolarization Factors $(\mathrm{A})^{a}$

\begin{tabular}{llll} 
model (eq) & pol & \multicolumn{1}{c}{$\mathbf{A}$} & $f(\mathbf{A})$ \\
Max.-Garn. (1) & TM & $1 / 3$ & 2.02 \\
Brugg. (2) & & $1 / 3$ & 0.28 \\
Brugg (3) & & {$[0.66,0.34,0]$} & 0.12 \\
Max-Garn (1) & TE & $1 / 3$ & 0.34 \\
Brugg (2) & & $1 / 3$ & 0.08 \\
Brugg (3) & & {$[0.66,0.34,0]$} & 0.04
\end{tabular}

${ }^{a} \mathrm{~A}$ lower value of cost function indicates a better fit.

$A_{3}=0$ ). This would not in general be the case (e.g., for an anisotropic sample). Both fits yield $A_{3}=0$, which thus selects a wire (i.e., a uniaxially infinitely extended ellipsoid) with an elliptical cross-section. It is therefore clear that the microscopically anisotropic Bruggeman model consisting of randomly oriented wire inclusions is the best of the three effective medium models considered and that only a single set of 
depolarization factors, $A_{1}=0.66, A_{2}=0.34$, and $A_{3}=0$, is appropriate to model both TM and TE measurements.

A good model for the effective permittivity of the gyroid metamaterial should be capable of reproducing not only the measured reflectance spectra presented in this work but also the previously reported behaviors as a function of refractive index of the host and volume fill fraction of gold. Figure 4 compares measurements performed by Salvatore et al. on 30\% fill fraction gold gyroids infiltrated with various refractive index media with the optimized Bruggeman model. ${ }^{12}$ Following the convention in that work, each normal incidence spectrum is characterized by its plasma edge, the wavelength of the point of inflection of the reflectance spectrum. In the simplest Drude model of the permittivity of metals, the plasma wavelength is the wavelength at which the permittivity changes from positive to negative and is characteristic of the density and effective mass of the free electrons in the particular metal. The plasma edge is not the plasma wavelength but behaves similarly and may be more readily and systematically identified from experimental data than the plasma wavelength (see for example Figure S6 of ref 12). Figure $4 \mathrm{a}$ reproduces the reflectance spectra measured by Salvatore et al. upon infiltration of the gyroid metamaterial with media of refractive indices $n_{\text {fill }}=1.0,1.33,1.5$, and 1.7 and indicates the plasma edge in each case (the labeled vertical lines). Note how the plasma edge is red-shifted with increasing host refractive index. Figure $4 \mathrm{~b}$ plots equivalent normal incidence reflectance spectra derived using the optimized Bruggeman model with $\varepsilon_{2}(\omega)$ of eq 3 set to $n_{\text {fill }}^{2}$, respectively. Again the plasma edge is red-shifted with increasing host permittivity. Although the measured and modeled plasma edges do not perfectly coincide, it is clear from Figure $4 c$ that the modeled plasma edges are within experimental error for each refractive index. Therefore, despite the dissimilarity between the measured and modeled normal incidence reflectance spectra, the model successfully predicts the plasma edge of the gold gyroid metamaterial. Furthermore, the optimized Bruggeman model performs better in this respect than the trihelical metamaterial analytical model, also reproduced from Salvatore et al. and shown in Figure 4c. ${ }^{12}$

The results of the optimized Bruggeman model may also be compared to previously measured data where the volume fill fraction of gold is varied. Figure 5a shows normal incidence reflectance spectra from Salvatore et al. as the volume fill fraction of the gold gyroid is increased from $30 \%$ to $90 \%$. Only four of the five plasma edges are shown, since those for the $75 \%$ and $90 \%$ fill fraction gyroids are within $2 \mathrm{~nm}$ of one another. Whereas the plasma edge is red-shifted with increasing refractive index of the host, it is blue-shifted as the fill fraction increases and the reflectance of the gyroid metamaterial tends toward that of bulk gold. Figure $5 \mathrm{~b}$ plots equivalent normal incidence reflectance spectra calculated from the optimized Bruggeman model. Here only two of the five plasma edges are shown, as all of those corresponding to fill fractions above $30 \%$ are extremely close (within $2 \mathrm{~nm}$ ). Again the plasma edge is blue-shifted with increased fill fraction, as it was in the measured spectra. However, as can be seen in Figure 5c, there is significant deviation between the measured and predicted plasma edges for intermediate fill fractions between about $40 \%$ and $70 \%$. Outside of this region (i.e., at low and high fill fractions) the optimized Bruggeman model performs well and is comparable with the THM model.

\section{DISCUSSION}

The failure of the Maxwell-Garnett theory to describe multidomain gyroid samples is perhaps not surprising, since its validity is limited to a low volume fraction of spherical dielectric inclusions that are spatially separated. The gyroid samples considered here consist however of an interconnected, percolating network of gold struts.

The better description of the experimental system by the Bruggeman model with spherical inclusions arises primarily from the fact that this model retains it validity for a high density of scatterers, expressed in the model by the fill fraction. The results of Figure 3 indicate that a model based on an effective medium approach is able to approximate the random multidomain gyroid network, despite the morphological dissimilarity.

While a model with a larger number of free parameters (i.e., the three depolarization factors) is expected to improve the level of agreement with the data, it is revealing that fits of both reflected TE and TM polarized light result always in zero values for one of the depolarization factors $A_{k}$. Since $A_{k}<1 / 3$ corresponds to a prolate ellipse, with a divergence for $A_{k} \rightarrow 0$, the optimization of the Bruggeman model with free $A_{k}$ values results in an effective medium consisting of randomly oriented wires of elliptical cross-section (see Figure 6 inset). While a random wire assembly is a better description than isolated individual scatterers for an interconnected gyroid network, the good agreement between fit and data is nevertheless surprising given that the Bruggeman theory does not model well percolating networks. ${ }^{19}$ Although allowing for microscopic anisotropy within the Maxwell-Garnett model (i.e., ellipsoidal inclusions) might similarly improve upon the results of the equivalent model with spherical inclusions, this variant of the Maxwell-Garnett model would still suffer from a limited validity because of the high volume fill fractions of our samples and was therefore not considered.

Like the classical wire-grid metamaterial, the gold gyroid metamaterial is shown to exhibit a strongly red-shifted plasma edge. ${ }^{20}$ The effective permittivity $\varepsilon_{\text {eff }}(\omega)$ derived from the Bruggeman theory with optimized depolarization factors, shown in Figure 6a, has a crossover of the real part of the permittivity just below $650 \mathrm{~nm}$ (arrow). In contrast, the real part of the permittivity of the constituent gold does not cross the zero axis at any wavelength in the visible (inset). Notwithstanding the effect of losses associated with the imaginary part of the permittivity, the gold gyroid metamaterial therefore behaves as both a dielectric $(\lambda \lesssim 650 \mathrm{~nm})$ and metal $(\lambda \gtrsim 650 \mathrm{~nm})$ in the visible. In the simple Drude model of the permittivity of a metal, this crossover is associated with the plasma frequency (plasma wavelength), an association that is hard to isolate in more complicated models (e.g., DrudeLorentz) or experimentally measured permittivities. The previously introduced plasma edge is thus useful to characterize the optical response of the gyroid metamaterial, despite the lack of a direct correlation with prominent features in the permittivity plots. The variation of the predicted real part of the permittivity with refractive index of the host and fill fraction of gold is shown in Figure $6 \mathrm{~b}$ and $\mathrm{c}$, respectively. Although quantitatively distinct, it is clear that the general behaviors of both the plasma edge (Figures $4 \mathrm{c}$ and $5 \mathrm{c}$ ) and the crossover wavelength (Figure $6 \mathrm{~b}$ and $\mathrm{c}$ ) are broadly similar. On the basis of this and the good fit of the Bruggeman model to the specular reflectance spectra, we conclude that gyroid metamaterials 

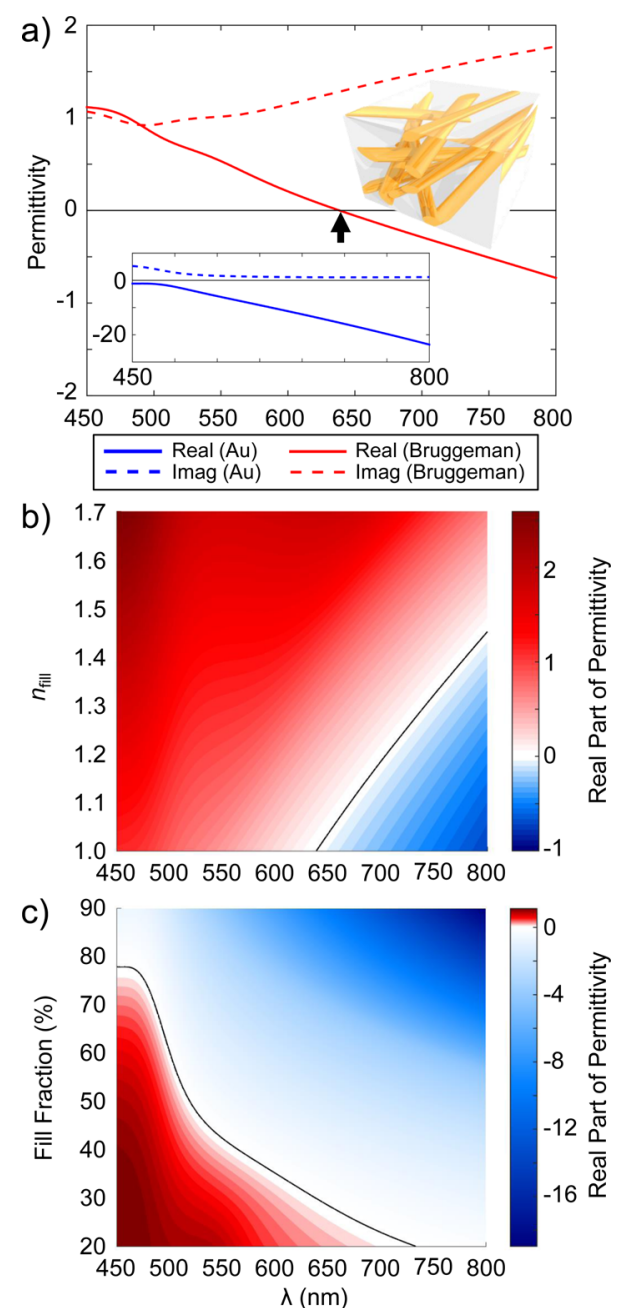

Figure 6. Effective permittivity of small domain gyroid metamaterials derived from the Bruggeman effective medium theory. (a) Real (solid line) and imaginary (dashed line) parts of the effective permittivity $\varepsilon_{\text {eff }}(\omega)$ calculated using the Bruggeman theory by setting $\varepsilon_{1}(\omega)=$ $\varepsilon_{\mathrm{Au}}(\omega), \varepsilon_{2}(\omega)=1, f_{1}=0.3, A_{1}=0.66, A_{2}=0.34$, and $A_{3}=0$ in eq 3 . The insets are a sketch of the equivalent structure corresponding to the optimized Bruggeman model and the real (solid line) and imaginary (dashed lines) part of the permittivity of bulk gold $\varepsilon_{\mathrm{Au}}(\omega)$. (b) Variation of the real part of the effective permittivity with refractive index of the host medium. (c) Variation of the same with volume fill fraction of gold.

averaged over many randomly oriented domains behave similarly to amorphous composites of air and gold (i.e., dilute metals) and therefore possess a similar response to nanoporous gold fabricated, for example, by dealloying. ${ }^{21-28}$

Although the optimized Bruggeman model is successful in predicting the optical properties of multidomain gold gyroid optical metamaterials (allowing to conclude that their optical properties are similar to nanoporous gold), it is by no means perfect, and various dissimilarities exist between the measured and predicted spectra. Surprisingly, the measured reflectance spectra are generally higher than those predicted. This is most likely caused by the details of the gyroid surface. For example, surface reconstruction (i.e., local reorientation) of the terblock terpolymer during thermal annealing can alter the in-coupling of light into the gyroid network. Alternatively, the details of the gyroid fabrication process might affect the surface roughness and the volume fill fraction of gold near the top surface, accounting for the differences in the reflectivity.

The relative decrease in the long-wavelength reflectance at high angles of incidence predicted by the Bruggeman model, more prominent when spherical inclusions are assumed and not present in the data, may similarly be a result of the gyroid metamaterial having a somewhat larger volume fill fraction of gold at its surface compared with that expected from a planar cut across the gyroid morphology. Incorrect fill fractions during optimization of the depolarization factors may also help to explain the relative inaccuracy of the model at intermediate fill fractions (i.e., between about $40 \%$ and $70 \%$ ) compared to the relative accuracy of the same model with respect to increasing refractive index of the host. However, to note that the fabrication of gyroid metamaterials with increased fill fraction and the accurate measurement of the fill fraction are difficult compared to infiltration of the metamaterial with varying refractive index media. ${ }^{12}$ Results of the former experiments should therefore be treated with some caution.

Finally, whereas all of the effective medium models considered are insensitive to the absolute size of the subwavelength inclusions, it is known that the optical properties of gold gyroid metamaterials vary with unit cell size. ${ }^{12}$ The results presented here are therefore limited to gold gyroids with a similar unit cell size (i.e., $\sim 60 \mathrm{~nm}$ ), although the methodology can of course easily be extended to gold gyroids of other unit cell sizes. Despite the potential shortcomings of the relatively simple analytical models employed here, the application of more complicated analytical models would not alter the current conclusion: that multidomain gold gyroid optical metamaterials behave substantially as if they were nanoporous gold.

\section{CONCLUSION}

In this work, the effective permittivity of multidomain gold gyroid metamaterials was estimated by comparing the results of three effective medium theories to measured specular reflectance spectra over a range of angles of incidence. We show that a microscopically anisotropic Bruggeman theory with wire inclusions provides the best fit to the data and that the optimized depolarization factors imply a composite of randomly oriented gold wires of elliptical cross-section. That the averaged optical properties of multidomain gyroid metamaterials are well described by an effective medium theory, including their variation with refractive index of the host and volume fill fraction of gold, contradicts previous assertions that the subwavelength structure necessarily alone distinguishes gold gyroid metamaterials from amorphous nanoporous gold. ${ }^{11}$

This result is surprising, given the experimental results obtained for large individual gyroid domains. ${ }^{11}$ In particular, the gyrotropic transmission along one of the chiral gyroid axes that was attributed to the self-inductance of plasmon-polariton modes is not captured by the effective medium model. If symmetry-related effects of the intricate gyroid structure do indeed contribute to the optical properties of a macroscopic sample consisting of numerous randomly oriented domains, it seems that this contribution is averaged out through the random alignment of the multidomain gyroid assembly, as also appears to be the case for gyroid photonic crystals. ${ }^{29,30}$ 


\section{EXPERIMENTAL METHODS}

Fabrication. Gold gyroid metamaterial fabrication has been detailed elsewhere. ${ }^{11-13,31}$ In brief, samples are prepared using substrates of fluorine-doped tin oxide (FTO)-coated glass (Solaronix) that have been cleaned using a piranha etch and immersed for $15 \mathrm{~s}$ in a $0.2 \%$ solution $(\mathrm{v} / \mathrm{v})$ of octyltrichlorosilane (Sigma-Aldrich) in anhydrous cyclohexane (SigmaAldrich). An $80 \mathrm{~kg} / \mathrm{mol}$ polyisoprene- $b$-polystyrene- $b$-poly(ethylene oxide) triblock terpolymer with block volume fractions of $30 \%, 53 \%$, and $17 \%$, respectively (synthesized by anionic polymerization as detailed elsewhere $\left.{ }^{32,33}\right)$, is spun from a $10 \%(\mathrm{w} / \mathrm{w})$ anhydrous anisole (Sigma-Aldrich) solution at $1200 \mathrm{rpm}$, resulting in an approximately $1 \mu \mathrm{m}$ thick ISO film as measured by thin film interferometry. Samples are subsequently thermally annealed under vacuum at $180{ }^{\circ} \mathrm{C}$ for $30 \mathrm{~min}$ (ramp rate $150{ }^{\circ} \mathrm{C} / \mathrm{h}$ ), then allowed to cool over approximately $12 \mathrm{~h}$. The polyisoprene block is degraded by exposure to UV light (VWR International, $254 \mathrm{~nm}, \sim 50 \mathrm{~mW} / \mathrm{cm}^{2}$ ) for up to $2 \mathrm{~h}$ and removed by dissolution in ethanol. The resulting template is filled by electrodeposition of gold (Metalor ECF60 and 0.5\% $(\mathrm{v} / \mathrm{v})$ E3 brightener) at a constant potential of $-0.8 \mathrm{~V}$ following a nucleation step (a cyclic voltammetry scan at a rate of $0.05 \mathrm{~V} / \mathrm{s}$ with vertex potentials of $0,-1.2$, and $0 \mathrm{~V}$ ). The three-electrode cell consisted of the FTO-coated glass substrate as the working electrode, an evaporated gold thin film as the counter electrode, and a saturated calomel reference electrode. Gold is grown both within and atop the polymer template, and the layer of excess bulk gold is cleaved at the interface between the nanostructured and bulk gold using Kapton tape, to ensure a smooth top surface (i.e., low surface roughness). Following electrodeposition, the remaining polymer template was removed by exposure to oxygen plasma for approximately 10 min (Diener MRC 100 at 100\% power). The resulting gold single gyroid has a unit cell size of $\sim 60 \mathrm{~nm}$ and a fill fraction of $30 \%{ }^{11,12}$

Characterization. Optical characterization is performed using an automated reflection goniometer with a fixed illumination arm (QP600-2-SR-BX $600 \mu \mathrm{m}$ core fiber and HPX-2000 Ocean Optics xenon light source). The sample and detection arm are arranged in the $\theta-2 \theta$ configuration such that whenever the sample is tilted by an angle $\theta$, the detection arm is tilted by an angle $2 \theta$. The spectra are recorded by coupling the reflected light into an optical fiber (QP230-2-XSR $230 \mu \mathrm{m}$ core) connected to a spectrometer (Ocean Optics QE6500) and are normalized at all angles of incidence with respect to the reflectance of a silver mirror. Linear polarizers are placed at the output of the illumination fiber and at the input of the detection fiber, fixed to the illumination and detection arms, respectively. Spectra are recorded at angles of incidence between $15^{\circ}$ and $65^{\circ}$ in steps of $1^{\circ}$. The procedure is repeated for both TE and TM polarizations in parallel configurations (i.e., aligned polarizer and analyzer).

Model. Where an effective medium model requires fitting, the MATLAB optimization toolbox is used to minimize the cost function:

$$
\begin{aligned}
& f(\mathbf{A})=\frac{1}{N \times M} \sum_{i=1}^{N} \times \\
& \sum_{j=1}^{M}\left(\log _{10} R_{\exp }\left(\lambda_{i}, \theta_{j}^{\text {in }}\right)-\log _{10} R_{\text {model }}\left(\lambda_{i}, \theta_{j}^{\text {in }}, \mathbf{A}\right)\right)^{2}
\end{aligned}
$$

such that

$$
A_{1}+A_{2}+A_{3}=1
$$

where $\mathbf{A}=\left[A_{1}, A_{2}, A_{3}\right], A_{k}$ is the $k$ th depolarization factor, $\lambda_{i}$ is the $i$ th wavelength, $\theta_{j}^{\text {in }}$ is the $j$ th angle of incidence, $N$ is the total number of wavelengths, $M$ is the total number of angles of incidence, and $R_{\text {exp }}\left(\lambda_{i}, \theta_{j}^{\text {in }}\right)$ and $R_{\text {model }}\left(\lambda_{i}, \theta_{j}^{\text {in }}, \mathbf{A}\right)$ are the experimental and modeled reflectance spectra, respectively. The modeled reflectance spectra are in turn calculated from the Fresnel equations for reflection from a semi-infinite nonmagnetic homogeneous medium for TE:

$$
R_{\text {model }}^{\mathrm{TE}}\left(\lambda_{i}, \theta_{j}^{\text {in }}, \mathbf{A}\right)=\left|\frac{n_{\text {air }} \cos \left(\theta_{j}^{\text {in }}\right)-n_{\text {eff }}\left(\lambda_{i}, \mathbf{A}\right) \cos \left(\theta_{j}^{\mathrm{tr}}\right)}{n_{\text {air }} \cos \left(\theta_{j}^{\text {in }}\right)+n_{\text {eff }}\left(\lambda_{i}, \mathbf{A}\right) \cos \left(\theta_{j}^{\text {tr }}\right)}\right|^{2}
$$

and TM:

$$
R_{\text {model }}^{\mathrm{TM}}\left(\lambda_{i}, \theta_{j}^{\text {in }}, \mathbf{A}\right)=\left|\frac{n_{\text {eff }}\left(\lambda_{i}, \mathbf{A}\right) \cos \left(\theta_{j}^{\text {in }}\right)-n_{\text {air }} \cos \left(\theta_{j}^{\text {tr }}\right)}{n_{\text {eff }}\left(\lambda_{i}, \mathbf{A}\right) \cos \left(\theta_{j}^{\text {in }}\right)+n_{\text {air }} \cos \left(\theta_{j}^{\text {tr }}\right)}\right|^{2}
$$

polarized light, where $n_{\text {air }}$ and $n_{\text {eff }}\left(\lambda_{i}, \mathbf{A}\right)$ are the refractive indices of air and the effective medium respectively, and $\theta_{j}^{\mathrm{tr}}$ is the $j$ th (complex) transmitted angle calculated by Snell's law, i.e.,

$$
n_{\mathrm{air}} \sin \left(\theta_{j}^{\text {in }}\right)=n_{\text {eff }}\left(\lambda_{i}, \mathbf{A}\right) \sin \left(\theta_{j}^{\text {tr }}\right)
$$

The assumption that the gold gyroid metamaterial appears semi-infinite (and that the derived effective permittivity is insensitive to film thickness) is based upon the thickness of the film $(\sim 1 \mu \mathrm{m})$, the unit cell size $(\sim 60 \mathrm{~nm})$, and previous studies of film thickness effects in gold gyroid optical metamaterials, ${ }^{34}$ which indicate that the reflectance spectra do not change beyond a threshold sample thickness. The effective medium models from which $n_{\text {eff }}\left(\lambda_{i}, \mathbf{A}\right)$ is derived require as an input the permittivity of bulk gold, $\varepsilon_{\mathrm{Au}}(\omega)$, which is here taken as that measured by Olmon et al. ${ }^{35}$ Optimized depolarization factors are calculated for each polarization state of light.

\section{ASSOCIATED CONTENT}

\section{Supporting Information}

The Supporting Information is available free of charge on the ACS Publications website at DOI: 10.1021/acsphotonics.6b00400. Additional data related to this publication is available at the University of Cambridge data repository (http://dx.doi.org/10.17863/CAM.4376).

Supplementary Figures S1-S3 and details on the derivation of the Bruggeman effective medium theory (PDF)

\section{AUTHOR INFORMATION}

\section{Corresponding Author}

*E-mail: bodo.wilts@unifr.ch. Phone: +41 (0)26 3008969.

\section{Present Addresses}

$\square$ Papierfabrik Louisenthal GmbH, 83701 Gmund a.T., Germany.

\#The Dow Chemical Company, 2301 N. Brazosport Boulevard, Freeport, Texas 77541, United States.

\section{Notes}

The authors declare no competing financial interest. 


\section{ACKNOWLEDGMENTS}

We thank Alexander Macfaden and Calum Williams for valuable comments on early versions of the manuscript. This research was supported through the Swiss National Science Foundation through the National Center of Competence in Research Bio-Inspired Materials and grant numbers 200021163220 (to U.S.) and PZ00P2 168223 (to B.D.W.), the Adolphe Merkle Foundation, the Engineering and Physical Sciences Research Council (EPSRC) through the Cambridge NanoDTC EP/G037221/1, EP/L027151/1, and EP/ G060649/1, and ERC LINASS 320503 and from the European Union's Horizon 2020 Research and Innovation Programme under the Marie Sklodowska-Curie grant agreement no. 706329 (to I.G.). Y.G. and U.W. thank the National Science Foundation (DMR-1409105) for financial support.

\section{REFERENCES}

(1) Cai, W.; Shalaev, V. Optical Metamaterials; Springer, 2010.

(2) Pendry, J. B. Negative refraction makes a perfect lens. Phys. Rev.

Lett. 2000, 85, 3966-9.

(3) Pendry, J. B.; Schurig, D.; Smith, D. R. Controlling Electromagnetic Fields. Science 2011, 1780, 1780-1782.

(4) Poddubny, A.; Iorsh, I.; Belov, P.; Kivshar, Y. Hyperbolic metamaterials. Nat. Photonics 2013, 7, 948-957.

(5) Boltasseva, A.; Shalaev, V. M. Fabrication of optical negativeindex metamaterials: Recent advances and outlook. Metamaterials 2008, 2, 1-17.

(6) Soukoulis, C. M.; Wegener, M. Past achievements and future challenges in the development of three-dimensional photonic metamaterials. Nat. Photonics 2011, 5, 523-530.

(7) Hur, K.; Francescato, Y.; Giannini, V.; Maier, S. A.; Hennig, R. G.; Wiesner, U. Three-dimensionally isotropic negative refractive index materials from block copolymer self-assembled chiral gyroid networks. Angew. Chem., Int. Ed. 2011, 50, 11985-11989.

(8) Dolan, J. A.; Wilts, B. D.; Vignolini, S.; Baumberg, J. J.; Steiner, U.; Wilkinson, T. D. Optical Properties of Gyroid Structured Materials: From Photonic Crystals to Metamaterials. Adv. Opt. Mater. 2015, 3, 12-32.

(9) Hsueh, H.-Y.; Yao, C.-T.; Ho, R.-M. Well-ordered nanohybrids and nanoporous materials from gyroid block copolymer templates. Chem. Soc. Rev. 2015, 44, 1974-2018.

(10) Wu, L.; Zhang, W.; Zhang, D. Engineering Gyroid-Structured Functional Materials via Templates Discovered in Nature and in the Lab. Small 2015, 11, 5004-5022.

(11) Vignolini, S.; Yufa, N. A.; Cunha, P. S.; Guldin, S.; Rushkin, I.; Stefik, M.; Hur, K.; Wiesner, U.; Baumberg, J. J.; Steiner, U. A 3D optical metamaterial made by self-assembly. Adv. Mater. 2012, 24, OP23-7.

(12) Salvatore, S.; Demetriadou, A.; Vignolini, S.; Oh, S. S.; Wuestner, S.; Yufa, N. A.; Stefik, M.; Wiesner, U.; Baumberg, J. J.; Hess, O.; Steiner, U. Tunable 3D Extended Self-Assembled Gold Metamaterials with Enhanced Light Transmission. Adv. Mater. 2013, 25, 2713-2716.

(13) Salvatore, S.; Vignolini, S.; Philpott, J.; Stefik, M.; Wiesner, U.; Baumberg, J. J.; Steiner, U. A high transmission wave-guide wire network made by self-assembly. Nanoscale 2014, 7, 2040-3372.

(14) Demetriadou, A.; Hess, O. Analytic theory of optical nanoplasmonic metamaterials. Phys. Rev. B: Condens. Matter Mater. Phys. 2013, 87, 161101.

(15) Oh, S. S.; Demetriadou, A.; Wuestner, S.; Hess, O. On the origin of chirality in nanoplasmonic gyroid metamaterials. Adv. Mater. 2013, 25, 612-7.

(16) Bergman, D. J.; Stroud, D. Physical Properties of Macroscopically Inhomogenous Media. Solid State Phys. 1992, 46, 147-269.

(17) Gaponenko, S. V. Introduction to Nanophotonics; Cambridge University Press: Cambridge, 2010.

(18) Bánhegyi, G. Colloid Polym. Sci. 1986, 264, 1030-1050.
(19) Kirkpatrick, S. Percolation and conduction. Rev. Mod. Phys. 1973, 45, 574.

(20) Pendry, J. B.; Holden, A. J.; Stewart, W. J.; Youngs, I. Extremely low frequency plasmons in metallic mesostructures. Phys. Rev. Lett. 1996, 76, 4773-4776.

(21) Yu, F.; Ahl, S.; Caminade, A.-M.; Majoral, J.-P.; Knoll, W.; Erlebacher, J. Simultaneous excitation of propagating and localized surface plasmon resonance in nanoporous gold membranes. Anal. Chem. 2006, 78, 7346-50.

(22) Dixon, M. C.; Daniel, T. A.; Hieda, M.; Smilgies, D. M.; Chan, M. H. W.; Allara, D. L. Preparation, structure, and optical properties of nanoporous gold thin films. Langmuir 2007, 23, 2414-22.

(23) Maaroof, A. I.; Gentle, A.; Smith, G. B.; Cortie, M. B. Bulk and surface plasmons in highly nanoporous gold films. J. Phys. D: Appl. Phys. 2007, 40, 5675-5682.

(24) Biener, J.; Nyce, G. W.; Hodge, A. M.; Biener, M. M.; Hamza, A. V.; Maier, S. A. Nanoporous Plasmonic Metamaterials. Adv. Mater. 2008, 20, 1211-1217.

(25) Sardana, N.; Birr, T.; Schlenker, S.; Reinhardt, C.; Schilling, J. Surface plasmons on ordered and bi-continuous spongy nanoporous gold. New J. Phys. 2014, 16.06305310.1088/1367-2630/16/6/063053

(26) Sardana, N.; Heyroth, F.; Schilling, J. Propagating surface plasmons on nanoporous gold. J. Opt. Soc. Am. B 2012, 29, 1778.

(27) Lang, X.; Qian, L.; Guan, P.; Zi, J.; Chen, M. Localized surface plasmon resonance of nanoporous gold. Appl. Phys. Lett. 2011, 98, 2009-2012.

(28) Maaroof, A. I.; Cortie, M. B.; Smith, G. B. Optical properties of mesoporous gold films. J. Opt. A: Pure Appl. Opt. 2005, 7, 1464-4258.

(29) Michielsen, K.; De Raedt, H.; Stavenga, D. G. Reflectivity of the gyroid biophotonic crystals in the ventral wing scales of the Green Hairstreak butterfly, Callophrys rubi. J. R. Soc., Interface 2010, 7, 765771.

(30) Saba, M.; Wilts, B. D.; Hielscher, J.; Schröder-Turk, G. E. Absence of Circular Polarisation in Reflections of Butterfly Wing Scales with Chiral Gyroid Structure. Mater. Today: Proc. 2014, 1, 193208.

(31) Farah, P.; Demetriadou, A.; Salvatore, S.; Vignolini, S.; Stefik, M.; Wiesner, U.; Hess, O.; Steiner, U.; Valev, V. K.; Baumberg, J. J. Ultrafast Nonlinear Response of Gold Gyroid Three-Dimensional Metamaterials. Phys. Rev. Appl. 2014, 2, 044002.

(32) Bailey, T. S.; Pham, H. D.; Bates, F. A. Morphological Behavior Bridging the Symmetric $\mathrm{AB}$ and $\mathrm{ABC}$ States in the Poly(styrene-bisoprene-b-ethylene oxide) Triblock Copolymer System. Macromolecules 2001, 34, 6994-7008.

(33) Bailey, T. S.; Hardy, C. M.; Epps, T. H.; Bates, F. S. A Noncubic Triply Periodic Network Morphology in Poly(isoprene-b-styrene-bethylene oxide) Triblock Copolymers. Macromolecules 2002, 35, $7007-7017$

(34) Salvatore, S. Optical Metamaterials by Block Copolymer SelfAssembly; Springer International Publishing, 2015.

(35) Olmon, R. L.; Slovick, B.; Johnson, T. W.; Shelton, D.; Oh, S.H.; Boreman, G. D.; Raschke, M. B. Optical dielectric function of gold. Phys. Rev. B: Condens. Matter Mater. Phys. 2012, 86, 235147. 